\title{
Zu abgehoben!(?)
}

\author{
Jochen Schiewe $^{1}$
}

Published online: 31 August 2021

(c) The Author(s) 2021

„Die wissenschaftlichen Beiträge in den $\mathrm{KN}$ sind mir zu abgehoben! Und dann sind die auch noch fast alle in englischer Sprache."

Solche Aussagen hören die Schriftleitung der KN und der Vorstand der DGfK immer mal wieder. Ist die Kluft zwischen „Wissenschaftlerinnen und Wissenschaftlern“ und „Praktikerinnen und Praktikern“ in der Kartographie tatsächlich so groß? Und wenn ja: Ist das erst in den letzten Jahren passiert oder eine Aussage, die über Generationen hinweg immer wieder auftritt?

Über die Verwendung der englischen Sprache soll an diese Stelle nicht geschrieben werden-hierzu möchte ich auf eine frühere Kolumne aus dem Jahr 2019 (KN 69(4): A52-A53) verweisen. Lassen Sie uns stattdessen einmal einen Blick zurück auf die Themen werfen, die vor einer bzw. zwei Generationen in den wissenschaftlichen Beiträgen der KN behandelt wurden.

Vor zwei Generationen bzw. 50 Jahren—im Jahr 1971— befassten sich die meisten Beiträge mit spezifischen Kartenprodukten wie topographischen, Boden-, Baugrund- oder Stadtkarten. Es gab auch einen Grundlagenbeitrag von Ulrich Freitag zum (auch heute noch eher stiefmütterlich behandelten) Thema „Semiotik und Kartographie“ (KN 21(5): 171-182). Und schließlich wurde über die ersten Erfahrungen mit der EDV berichtet: So behandelte Adolf Hanle das Thema „Die Lochkarte und ihre Anwendung im geographisch-kartographischen Arbeitsbereich“ (KN 21 (2): 58-62). Darüber hinaus schilderten Wolf-Dieter Rase und Thomas K. Peucker ihre Erfahrungen mit SYMAP, einem Programm, mit dem Karten zur Ausgabe auf einem Zeilendrucker hergestellt werden konnte (KN 21 (2): 50-58).

Vor einer Generation bzw. 25 Jahren —im Jahr 1996-gab es immer noch einige Beiträge zu speziellen Kartenarten bzw. -produkten (TK25, Alpen, Gletscher). Die knappe Mehrzahl der Veröffentlichungen (u. a. von Frank Dickmann,

Jochen Schiewe

jochen.schiewe@hcu-hamburg.de

1 Hamburg, Germany
Doris Dransch, Liqiu Meng, Peter Tainz \& Wilfried Weber) befasste sich aber bereits mit der digitalen Kartographie. Georg Gartner beschäftigte sich mit dem damals brandneuen „Internet für Kartographen“ (KN 46(5): 185-190).

Die Herstellung und Fortführung diverser topographischer und anderer Kartenprodukte ist also schon vor 25 Jahren weniger behandelt worden. Dieser Trend hat sich fortgesetzt—das Thema wird in wissenschaftlichen Institutionen heute kaum noch behandelt.

Die Artikel von 1971 und 1996 zur digitalen Kartographie, so aktuell und interessant sie zu der Zeit auch waren, haben heute mehr oder minder keine Bedeutung mehr. Zudem erinnere ich mich auch (zumindest an die Zeit um 1996 und knapp davor ...), dass diese Themen von vielen Kartographinnen und Kartographen eher kritisch und als wenig relevant angesehen wurden (was angesichts der kartographischen Qualität der Ausgaben wie von SYMAP und dem notwendigen Aufwand zur Herstellung auch nicht erstaunt). Bezieht man auch 2021 mit ein, kann man gut nachvollziehen, dass die Fortschritte in der digitalen Welt bzw. der digitalen Kartographie nicht linear verlaufen sind. Es ist inzwischen weniger eine Frage von Generationen als von Dekaden oder noch kürzeren Zeiträumen, in denen man immer wieder neue technische Entwicklungen berücksichtigen und sich neue Denkweisen aneignen muss. Es ist wenig gewagt zu behaupten, dass die aktuellen 2021er Trends wie Virtual und Augmented Reality, Big Data oder Künstliche Intelligenz und Deep Learning in zehn Jahren nicht mehr Schwerpunkte in KN-Heften sein werden.

Und noch eine weitere Beobachtung aus dem Vergleich der Beiträge von 1971, 1996 und 2021: Auch wenn die Schnittstellen zur Informatik und Geo(infor)matik immer größer geworden sind (und auch im Untertitel unserer Gesellschaft Einzug gehalten haben): In den (allermeisten) Artikeln geht es immer noch um die kartographische Kernaufgabe-raumzeitliche Informationen so aufzubereiten und zu präsentieren, dass sie für einen bestimmten Zweck verständlich werden sowie neues Wissen erzeugen oder zu guten Entscheidungen beitragen können. Diese Nutzungs- und Aufgabenorientierung wird heute sogar noch 
konsequenter verfolgt: Im Gegensatz zu früheren Artikeln kommt eine Autorin oder ein Autor im Jahr 2021 nicht mehr an dem empirischen Nachweis einer solchen Gebrauchstauglichkeit vorbei.

Diese Beobachtungen zum Wandel wissenschaftlicher Themen beschreiben sicherlich einen Teil der oben genannten Kluft zwischen Wissenschaft und Praxis. Wandel hat es immer gegeben-nur erfolgt er immer schneller, was das „Hinterherkommen“ (übrigens auch von Wissenschaftlerinnen und Wissenschaftlern!) immer schwieriger macht.

Was können wir also tun?

Dass die Herstellung konkreter Kartenprodukte in der Forschung kaum noch Beachtung findet, soll m. E. die Expertinnen und Experten aus der Praxis keinesfalls davon abhalten, über interessante Entwicklungen und Überlegungen zu berichten. Der Informations- und Praxisteil in den $\mathrm{KN}$ ist die perfekte Plattform hierfür (übrigens in deutscher Sprache). Nebenbei: Ich behaupte auch, dass sich alle Leserinnen und Leser wieder über Kartenbeilagen in den $\mathrm{KN}$ freuen würden. Und ich rufe die eher ,praktisch orientierten" Kartographinnen und Kartographen auf, die wissenschaftlichen Beiträge nicht kategorisch beiseite zu legen. Stellen Sie vielmehr Fragen an die Autorinnen und Autoren-aus eigener Erfahrung kann ich Ihnen bestätigen, dass konstruktive Rückmeldungen viel mehr Freude bereiten als die Stille der Leserschaft.

Aber auch die „Wissenschaftlerinnen und Wissenschaftler" können dazu beitragen, die erwähnte Kluft zu verringern: Schreiben Sie zumindest umfangreichere und verständlichere Zusammenfassungen Ihrer Fachartikel! Und lesen Sie die Beiträge im Informations- und Praxisteil sehr aufmerksam! Auch die Einordnung der aktuellen Trends und Artikel sollte breiteren Raum finden-nicht nur in den Editorials, sondern verstärkt auch durch Überblicksbeiträge.

Alle, die dieses Heft in der Hand halten, teilen ihre Begeisterung für die Kartographie und arbeiten an deren Kernaufgaben. Wir sollten nun „Praxis“ und „Wissenschaft“ (wenn es denn diese Kategorien streng genommen überhaupt gibt) wieder stärker zusammenzuführen und von unserem jeweiligen Wissen profitieren. Genau das ist die Idee der DGfK und der KN, in der deshalb seit Generationen-im Gegensatz zu den meisten anderen internationalen Fachzeitschriften zur Kartographie-Praxis- und wissenschaftliche Beiträge auch mit Absicht vereint werden.
Ich wünsche Ihnen eine anregende Lektüre.

Ihr.

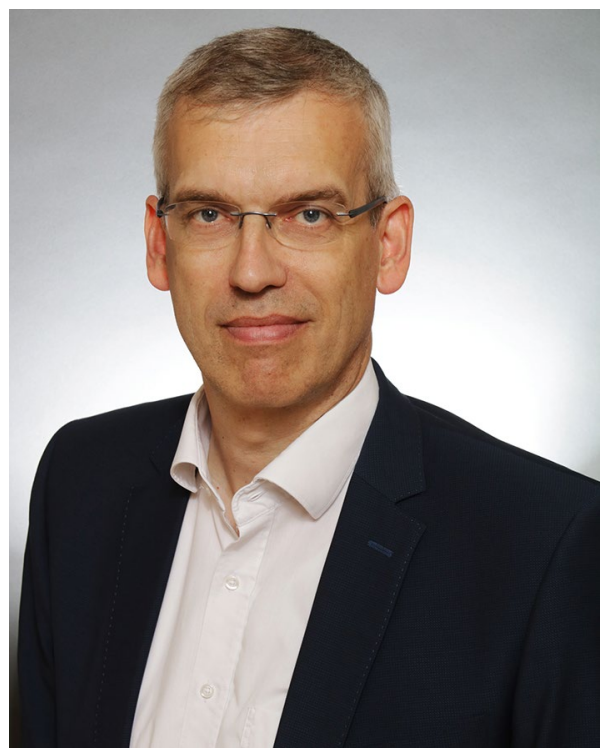

Jochen Schiewe.

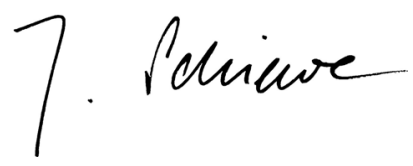

Supplementary Information The online version contains supplementary material available at https://doi.org/10.1007/s42489-021-00084-1.

Funding Open Access funding enabled and organized by Projekt DEAL.

Open Access This article is licensed under a Creative Commons Attribution 4.0 International License, which permits use, sharing, adaptation, distribution and reproduction in any medium or format, as long as you give appropriate credit to the original author(s) and the source, provide a link to the Creative Commons licence, and indicate if changes were made. The images or other third party material in this article are included in the article's Creative Commons licence, unless indicated otherwise in a credit line to the material. If material is not included in the article's Creative Commons licence and your intended use is not permitted by statutory regulation or exceeds the permitted use, you will need to obtain permission directly from the copyright holder. To view a copy of this licence, visit http://creativecommons.org/licenses/by/4.0/. 